\title{
Semi-artificial mouse skin membrane feeding technique for adult tick, Haemaphysalis longicornis
}

\author{
Takeshi Hatta', Takeharu Miyoshi', Makoto Matsubayashi', Md Khyrul Islam¹, M Abdul Alim¹, Anisuzzaman', \\ Kayoko Yamaji ${ }^{1}$, Kozo Fujisaki ${ }^{2}$ and Naotoshi Tsuji ${ }^{*}$
}

\begin{abstract}
Background: An in vitro artificial feeding technique for hard ticks is quite useful for studying the tick-pathogen interactions. Here, we report a novel semi-artificial feeding technique for the adult parthenogenetic tick, Haemaphysalis longicornis, using mouse skin membrane.

Findings: Skin with attached adult ticks was removed from the mouse body at 4 to 5 days post-infestation for the construction of the feeding system. This system supplied with rabbit blood was kept in $>95 \%$ relative humidity at $30^{\circ} \mathrm{C}$ during the feeding, and ticks were fully engorged (artificially engorged, AE) within 12 to $48 \mathrm{~h}$. For comparison, ticks were fed to engorgement solely on rabbit or mouse for 5 days as controls (naturally engorged on rabbit, NEr, or mouse, NEm). Blood digestion-related gene expression in the midgut and reproductive fitness were compared. Body weight, egg mass weight, egg conversion ratio, and hatchability of eggs did not show any significant differences. We analyzed transcription profiles of selected genes assayed by quantitative RT-PCR and revealed similar patterns of expression between NEr and AE but some differences between NEm and AE or NEm and NEr.

Conclusions: Our results demonstrate that this semi-artificial feeding technique mimics natural feeding processes of ticks and can be utilized as a standardized method to inoculate pathogens, especially Babesia protozoa, into $\mathrm{H}$. longicornis and possibly other tick species as well.
\end{abstract}

Keywords: Haemaphysalis longicornis, Mouse skin membrane system, Semi-artificial feeding technique, Tick

\section{Findings}

As vectors of pathogens, ticks transmit viruses, rickettsia and protozoan parasites to both animals and humans. Artificial feeding systems are attractive tools for investigating the mechanisms of pathogen transmission as well as for studying the tick-pathogen interactions. First, artificial feeding systems can reduce variation within a given treatment group because the blood meal is supplied from the same donor, which reduces the variation that arises from individual host-tick relationships [1]. Second, an animal experimental model is assumed to have a potent difficulty to control the infection in attached ticks with known numbers of pathogens, because pathogen load in ticks might be affected by the immune system of the hosts targeting tick molecules, such as protective

\footnotetext{
* Correspondence: tsujin@affrc.go.jp

'Laboratory of Parasitic Diseases, National Institute of Animal Health, National Agricultural and Food Research Organization, 3-1-5 Kannondai, Tsukuba, Ibaraki 305-0856, Japan

Full list of author information is available at the end of the article
}

antigen, subolesin [2]. In this model using artificial feeding, the effects of the host's immune responses against ticks are removed, and pathogens can be introduced into vectors in a controlled manner. So far, artificial feeding techniques have been used to feed a number of tick species of the family Ixodidae, including Rhipicephalus spp., Dermacentor spp., Amblyomma spp., Hyalomma spp., and Ixodes spp. using capillary tubes or membranes (briefly reviewed in [3]). Recently, Kröber and Guerin $[1,4,5]$ established a method using a silicone membrane to engorge Ixodes ricinus. Tajeri and Razmi [3] also attempted to use this membrane for Hy. anatolicum anatolicum and Hy. dromedarii. These tick species have a long hypostome and fine palps with a wide range of motion and can reasonably be expected to completely penetrate the artificial membrane.

On the other hand, Haemaphysalis longicornis, another ixodid tick, has a short hypostome and trigonal palps projecting laterally with very limited motion (Figure 1). Additionally, its hypostome and chelicerae are not thrust 


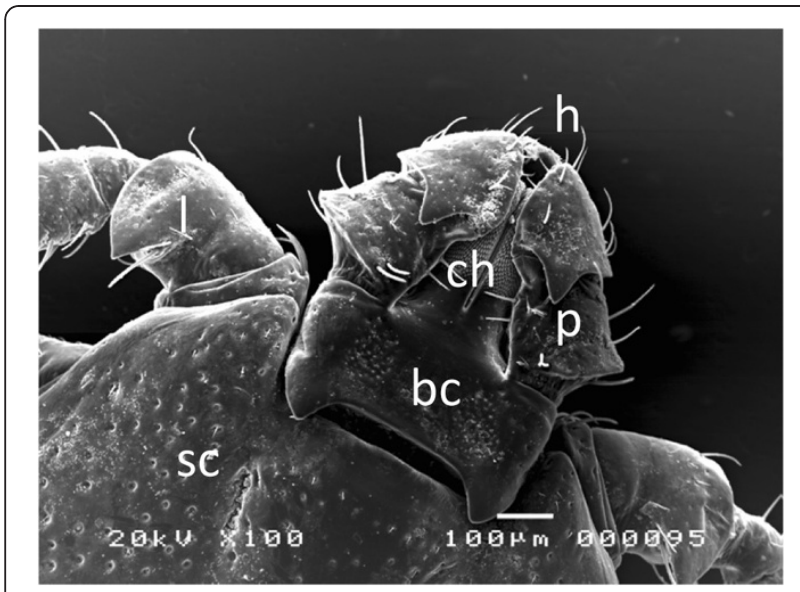

Figure 1 Capitulum of $\boldsymbol{H}$. longicornis. Scanning electron micrographs showing the mouthparts of $\mathrm{H}$. longicornis from the dorsal aspect. Bar $=100 \mu \mathrm{m}$. h, hypostome; ch, chelicera; p, palps; bc, basis capituli; sc, scutum; l, leg.

deeply into the host's dermis (Figure 2A) [6], therefore, it is quite difficult for $H$. longicornis to feed using capillary tube or silicone membrane-based in vitro feeding systems. To overcome these limitations, here, ticks were first fed on a mouse for 4 to 5 days, and then, the skin with the attached ticks was removed from the mouse for construction of a feeding system. This technique is a semi-artificial feeding technique and ticks were fed with supplied rabbit blood, and here, designated as artificially engorged (AE) post-engorgement phenotype, including blood feeding (engorgement body weight), reproductive fitness (egg mass weight and egg conversion ratio), and hatchability of eggs to larvae, as well as transcription profiles of selected genes expressed in midgut were compared to those of naturally engorged ticks fed on tick-naïve SPF rabbit (NEr) or mouse (NEm).

\section{Naturally engorged ticks}

The parthenogenetic Okayama strain of the tick H. longicornis has been maintained by feeding on rabbits in our laboratory since 1976 [7]. In this study, NEr ticks were prepared according to the usual method described previously [8]. Briefly, 20 adult ticks were placed on the ears of a rabbit to feed. At the beginning of the engorgement period, 9 ticks, which spontaneously detached from the rabbit after 5 days were collected. Of those, 6 randomly selected ticks were subjected to phenotypic analysis, and the remaining 3 ticks were used for transcription analysis. NEm ticks were also prepared according to the method described previously [9]. Briefly, 5 mice (BALB/ c, 3 weeks old) were infested with 10 adult ticks (2 ticks per mouse). Six randomly selected NEm ticks were subjected to phenotypic analysis, and the remaining 4 ticks were used for transcription analysis. Ethical approvals of conducting all animal experiments were provided by the Animal Care and Use Committee, National Institute of Animal Health (NIAH, Approval nos. 441 and 578).
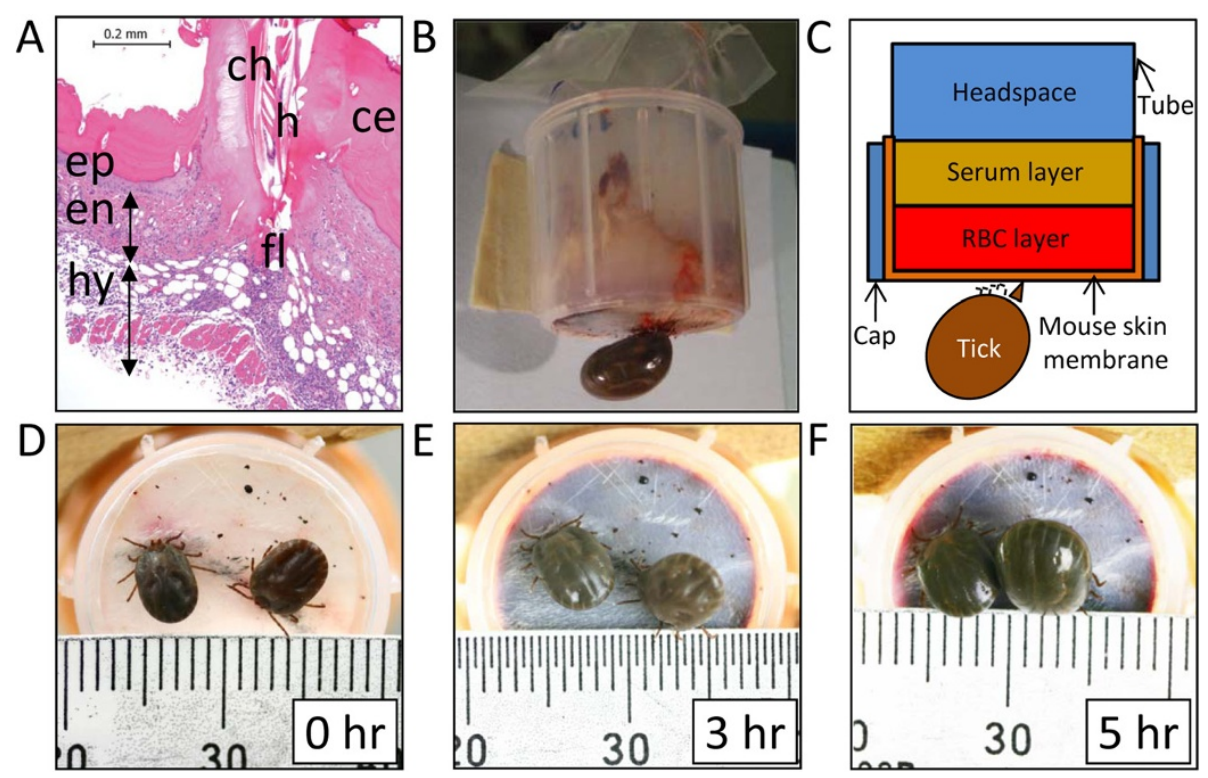

Figure 2 Mouse-skin membrane-based semi-artificial feeding device. (A) Tick bite lesion of mouse skin used as a membrane in semi-artificial feeding technique. h, hypostome; ch, chelicera; ce, cement; en, endodermis; ep, epidermis; hy, hypodermis; fl, feeding lesion. (HE, Bar: 200 um) (B) Lateral view of the semi-artificial feeding system with a feeding tick. (C) Schematic diagram of the system used in this study. (D-F) Chronological changes $(0,3,5$ h) of ticks feeding on the semi-artificial feeding system. 


\section{Artificially engorged ticks}

To prepare the mouse skin membrane, 5 to 7 adult ticks were allowed to feed on the shaven back of each of four tick-naïve SPF mice (BALB/c, 3 weeks old) following the described method [9]. After 4 to 5 days (at the beginning of the expansion period [10]), a rectangular section $\left(\sim 9 \mathrm{~cm}^{2}\right)$ of the mouse skin with the ticks attached was carefully removed from the mouse's body. Figure 2A shows the Hematoxylin and Eosin (HE)-stained lesion of mouse skin membrane used for the feeding system. Hypodermal layers around the feeding lesion were carefully removed from the skin with sterile tweezers as much as possible. The body of the feeding system (Figure 2B) was constructed using the upper part of a Falcon tube (\#352059, Becton, Dickinson and Company, Franklin Lakes, NJ) by cutting the tube at $\sim 3 \mathrm{~cm}$ from the top. Then, the roof of the cap of the Falcon tube was removed and the skin membrane with ticks was placed on the mouth of the tube keeping the ticks outside and held tightly in place by applying the cap. Only two ticks were selected and allowed to feed within the area of the skin membrane in this system, and the ticks in excess of two were removed by tweezers and weighed. The mean body weight of the removed ticks
$(24.9 \pm 5.2 \mathrm{mg})$ was subtracted from the weight of ticks after semi-artificial feeding to estimate weight gain. After construction, the inside of the membrane was washed with sterilized phosphate-buffered saline (PBS) supplemented with 100 units $/ \mathrm{ml}$ penicillin and $100 \mu \mathrm{g} / \mathrm{ml}$ streptomycin (Life Technologies Corporation, Carlsbad, $\mathrm{CA})$. Then, pre-warmed $\left(30^{\circ} \mathrm{C}\right)$ rabbit blood containing $300 \mu \mathrm{l}$ washed red blood cells (RBC) and $700 \mu \mathrm{l}$ sterile serum (filtered with a syringe filter; \#4652, $0.2 \mu \mathrm{m}$, Acrodisc Syringe Filters, Pall Co., Cornwall, UK.) was poured into the device (Figure 2C). To secure a sufficient volume of blood for the duration of tick feeding, we collected blood from a tick-naïve female SPF Japanese white rabbit (3- to 5-months-old). The open end of the tube was covered with a piece of parafilm. All procedures including system construction and blood exchange were performed inside a biosafety cabinet. The system was kept in a humidified chamber with $>95 \%$ relative humidity at $30^{\circ} \mathrm{C}$, and the rabbit blood was changed at every $12 \mathrm{~h}$. When partially fed ticks of the expansion period (4-5 days post-infestation, DPI) were used, fully engorged ticks dropped off within 12 to $48 \mathrm{~h}$ of the onset of artificial feeding (Figure 2D-F). In contrast, ticks in the late-growth phase (3 DPI) required feeding
A
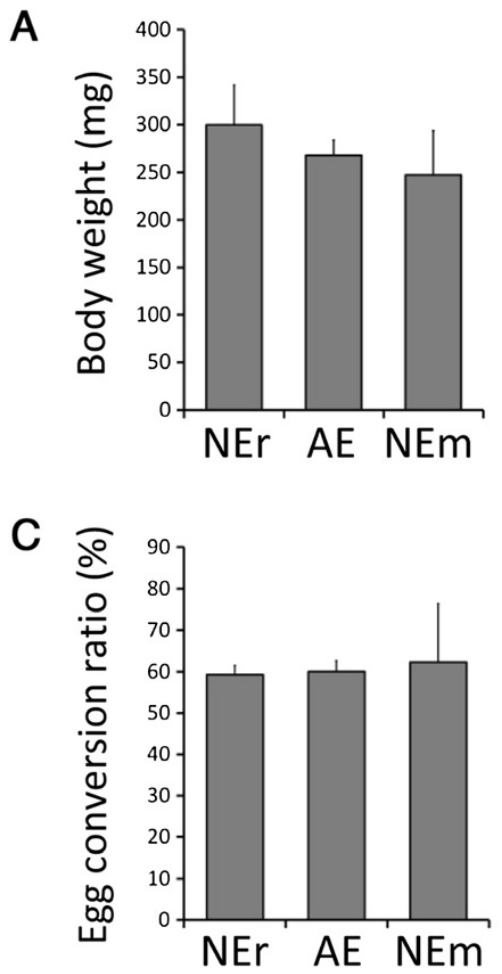

B
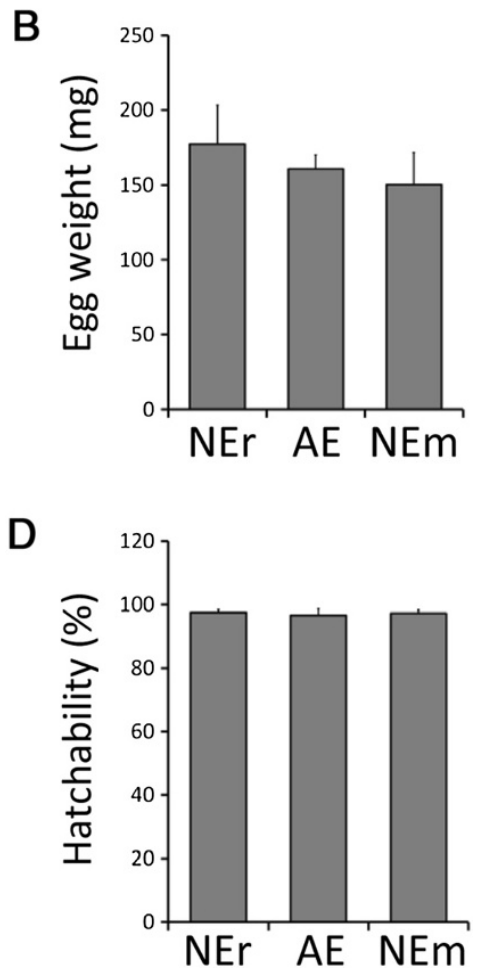

Figure 3 Phenotypic comparison of ticks fed on different feeding systems. (A) Engorged body weight of NEr $(n=6), \operatorname{AE}(n=4)$, and NEm $(n=6)$ ticks. The bars represent mean values and the error bars indicate the standard deviation. (B) Total egg mass weight measured at 10 DPO. (C) Egg conversion ratio (total egg weight/engorged body weight). (D) Comparison of hatchability of eggs (larval counts/total number of larvae and non-hatching eggs) derived from engorged ticks of different feeding systems. The results shown are from a single experiment and are representative of three independent experiments. 
on blood for more than $48 \mathrm{~h}$ to become engorged (data not shown). These feeding patterns were quite similar to the on-host feeding patterns of ticks described previously [10], suggesting that our in vitro feeding device effectively supports the expansion process of ticks and provides a sufficient amount of blood.

\section{Phenotypic analysis}

Blood feeding, reproductive parameters, and hatching rate of eggs were quantified to investigate the influences of artificial feeding on tick physiological phenotype. After repletion, the body weight gain of AE ticks was approximately 10 times that of their initial body weight. There were no significant differences in engorged body weight between $\mathrm{AE}$ and NEr or NEm ticks measured after spontaneous detachment of ticks following full engorgement (Figure 3A). After collection, the engorged ticks were placed separately in small sterile vials and incubated in a humidified chamber with $>95 \%$ relative humidity at $25^{\circ} \mathrm{C}$ for egg production. Almost all of the ticks started laying eggs at 4 to 5 days post-engorgement, however, ticks were monitored until 10 days post-oviposition (DPO) at which time point each egg mass obtained from individual ticks was collected separately and weighed. To determine hatching rates, the collected egg mass was placed separately in a paper envelope and incubated under the same conditions described above for 40 days until the eggs hatched. After hatching, larvae were counted manually and hatchability was estimated as described previously [11]. There were no significant differences between $\mathrm{AE}$ and $\mathrm{NEr}$ or NEm in terms of egg mass weight (Figure 3B), egg conversion ratio (total egg weight/engorged body weight, Figure 3C), or hatching rate (Figure 3D). In addition, larvae derived from the AE, NEr, and NEm lineages of ticks were able to feed normally on rabbit (data not shown).

\section{Transcription analysis}

We evaluated the effects of artificial feeding on the transcription of eight selected genes related to proteolysis of tick blood digestion in the midgut [12] such as $\mathrm{H}$. longicornis serine proteinase (HlSP) [GenBank:AB127388] [13], Longepsin [GenBank:AB218595] [14], Longipain [GenBank:AB255051] [15], H. longicornis serine carboxypeptidase 1 (HlSCP1) [GenBank:AB287330] [16], $H$. longicornis legumain (HlLgm) [GenBank:AB279705] [17], HlLgm2 [GenBank:AB353127] [18], H. longicornis leucine aminopeptidase (HLAP) [GenBank:AB251945] [19], and HILAP2 (Hatta and Tsuji, unpublished data). Additionally, we checked one defensin gene related to tick innate immunity in the midgut [20], namely, H. longicornis midgut defensin (Hlgut-defensin) [GenBank: EF432731] to judge the microbial contamination or infection in $\mathrm{AE}$ ticks since the transcription of this gene is drastically induced by lipopolysaccharide
(LPS) [21]. To do this, the midguts of NEr, AE, and NEm ticks were collected in sterile PBS at 3 days after repletion. Immediately after collection, the midguts were submersed into $3 \mathrm{ml}$ of Buffer RLT (RNeasy Mini Kit, Qiagen, Valencia, CA, USA) supplemented with $30 \mu \mathrm{l}$ of 2-mercaptoethanol, and thoroughly homogenized by passing the tissue mass through a 20-gauge needle (NN-2038R, Terumo, Tokyo, Japan) fitted to a $5 \mathrm{ml}$ syringe (SS-05LZ, Terumo) five times. Total RNA was isolated from the homogenates according to the manufacturer's instructions and used to synthesize cDNA (RNA PCR Kit Ver 3.0, Takara Bio INC., Shiga, Japan). Quantitative reverse transcription polymerase chain reaction (qRT-PCR) using SYBR Green I dye (LightCycler FastStart DNA Master SYBR Green I, Roche Diagnostics, Mannheim, Germany) and the primer sets listed in Table 1 was conducted to

Table 1 Primers for quantitative RT-PCR

\begin{tabular}{|c|c|}
\hline Gene name and primer ID & Primer sequences (5/ to $3 /$ ) \\
\hline \multicolumn{2}{|l|}{$\beta$-actin } \\
\hline HIActin8F1 & CCCATCTACGAGGGTTACGCTC \\
\hline HIActin9R1 & CATCTCCTGCTCGAAGTCCAGG \\
\hline \multicolumn{2}{|l|}{ HISP (serine protease) } \\
\hline HISPEf2 & TCCTTCCTCCTGAAGCAG \\
\hline HISPEr2 & CGTTCGCTATCCATGGTC \\
\hline \multicolumn{2}{|l|}{ Longepsin (aspartic protease) } \\
\hline longepsin_f2 & CATGAACGGCGTGAAAGTAG \\
\hline longepsin_r2 & TCCTTGCCTTCCAAAACG \\
\hline \multicolumn{2}{|l|}{ Longipain (cysteine protease) } \\
\hline longipain_f1 & ACCCTGCGACAAGAGCATAC \\
\hline longipain_r1 & TCCACTTGAATCTGCGTCAC \\
\hline \multicolumn{2}{|c|}{ HISCP1 (serine carboxypeptidase) } \\
\hline HISCP1_f1 & TGCTTCAGACTGCATTGACC \\
\hline HISCP1_r1 & TTGACCGCAGGTGTCATATC \\
\hline \multicolumn{2}{|l|}{ HILgm (legumain) } \\
\hline HLleg1RTf1 & CGACGAGCAAATCGTAGTCA \\
\hline HLleg1RTr1 & ACTITTCCGCTTCCTCCATT \\
\hline \multicolumn{2}{|l|}{ HILgm2 (legumain) } \\
\hline $\mathrm{B} 32 \mathrm{G} 12 \mathrm{f} 1$ & CCTTCGCAACAAGCTAAAGG \\
\hline B32G12r1 & TCAGAAGTCCTTCGGTGCTT \\
\hline \multicolumn{2}{|l|}{ HILAP (aminopeptidase) } \\
\hline HILAPf1 & CGCTAAGAAGCAGGCTGTCCTA \\
\hline HILAPr1 & TCAGACCGTAGAAAACTCTGGAC \\
\hline \multicolumn{2}{|l|}{ HILAP2 (aminopeptidase) } \\
\hline HILAP2f2 & AAGGCTCTTCACGAAGTGGA \\
\hline HILAP2r1 & TGGTCGACACCTCGAACATA \\
\hline \multicolumn{2}{|c|}{ Hlgut-defensin (anti-microbial peptide) } \\
\hline EF132731 F & GGGACTITTACTGGCTITCCTG \\
\hline EF132731 R & ACACGCCCTTTCATCGAAC \\
\hline
\end{tabular}




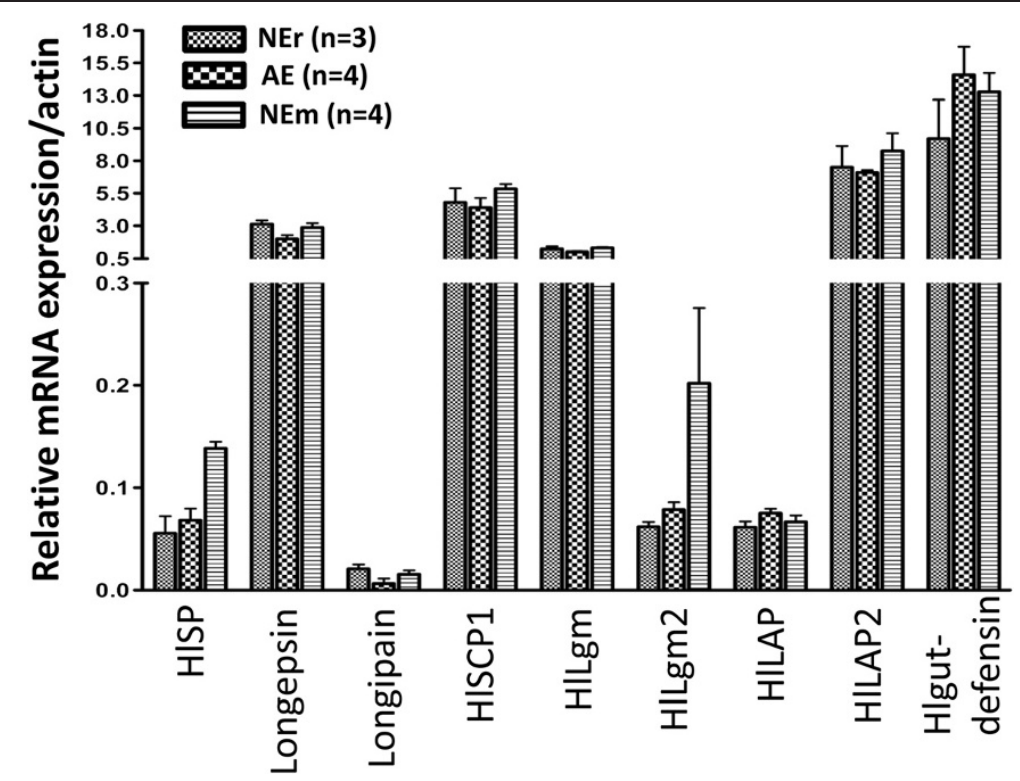

Figure 4 Comparison of transcriptional patterns of midgut-associated genes. Quantitative RT-PCR analysis of gene expression is presented relative to the internal standard $\beta$-actin and error bars represent the standard deviation. The results shown are from a single experiment and are representative of three independent experiments.

measure mRNA expression levels, as described previously [22]. Expression of each of the genes was normalized to that of $\beta$-actin [GenBank:AY254898] based on copy number (Figure 4). As expected, blood digestion-related gene expression patterns were quite similar between $\mathrm{AE}$ and NEr ticks, suggesting that the semi-artificial feeding technique does not affect midgut function parameters, such as blood uptake, expansion of midgut size, and blood-digestion. However, a slight difference in expression of two genes (HISP and HlLgm2) was observed in ticks of the feeding groups $\mathrm{AE}$ and NEm or of NEr and NEm, which may be due to the feeding of blood from different hosts, corroborating the findings of others [23].

Even though a biosafety cabinet was used throughout the study during construction and manipulation of the feeding device, the possibility of microbial contamination in the blood cannot be completely neglected since we did not use antibiotics in the supplied blood. Nevertheless, similar expression patterns of the Hlgut-defensin gene among AE, NEr, and NEm ticks indicate that the feeding system was quite free from bacterial contamination. However, to increase the certainty of avoiding bacterial contamination, it would be better to apply antibiotics to this feeding system for other applications.

\section{Conclusions}

In conclusion, our findings suggest that the semi-artificial feeding technique for $H$. longicornis is very effective and may be used for all tick species, especially those with short hypostomes that do not penetrate deeply in the dermal layer of the host, such as Rhipicephalus ticks [24].
Additionally, the technique is quite simple and cost effective since it does not require thinner artificial membrane [3], odorant (cow hair extracts), and/or feeding stimuli (adenosine triphosphate, ATP) $[1,4,5]$ to enhance tick attachment. Although it is necessary to sacrifice mice in order to construct the device, the technique bears great promise for conducting in vitro assays, including the inoculation of pathogens, especially Babesia protozoa, based on results reported by Callow [25] that Rhipicephalus (Boophilus) microplus tick infection with one of the tickborne bovine pathogens, Babesia bigemina, takes place during the rapid phase of feeding corresponding to the final stage (last $24 \mathrm{~h}$ ) of the blood feeding. We expect that this technique will be useful in studies of tick physiology, tick-pathogen interactions, and tick-host interactions regarding novel tick genes that respond to the host defense mechanisms such as coagulation, inflammation, and immune responses.

\section{Abbreviations}

AE: Artificially engorged; ATP: Adenosine triphosphate; DPI: Days postinfestation; DPO: Days post-oviposition; HE: Hematoxylin and eosin; HISP: H. longicornis serine proteinase; HISCP1: H. longicornis serine carboxypeptidase 1; HILgm: H. longicornis legumain; HILAP: H. longicornis leucine aminopeptidase; NE: Naturally engorged; NIAH: National Institute of Animal Health; PBS: Phosphate-buffered saline; RBC: Red blood cells; RT-PCR: Reverse transcription polymerase chain reaction.

Competing interests

The authors declare that they have no competing interests.

\section{Authors' contributions}

$\mathrm{TH}, \mathrm{NT}$, and KF were involved in the design of the experiments. TM, MAA and $M M$ carried out the rabbit experiment. A and KY carried out the mouse experiment. The manuscript was prepared by $\mathrm{TH}, \mathrm{MKI}$ and NT. All authors 
actively contributed to the interpretation of the findings, and read and approved the final manuscript.

\section{Acknowledgements}

This work was supported by the Grant-in-Aids (to TH, NT and KF) from the Ministry of Education, Culture, Sports, Science and Technology of Japan. This work was also supported by a grant (to NT and KF) for Promotion of Basic Research Activities for Innovative Biosciences from the Bio-oriented Technology Research Advancement Institution. We thank M. Shimada and M. Kobayashi for their generous help in preparing histological sections. We also thank Forte Science Communications (L. Gudex) for language editing of the manuscript.

\section{Author details}

${ }^{1}$ Laboratory of Parasitic Diseases, National Institute of Animal Health, National Agricultural and Food Research Organization, 3-1-5 Kannondai, Tsukuba, Ibaraki 305-0856, Japan. '2Laboratory of Emerging Infectious Diseases, School of Frontier Veterinary Medicine, Kagoshima University, Korimoto, Kagoshima 890-0065, Japan.

Received: 26 June 2012 Accepted: 11 October 2012

Published: 15 November 2012

\section{References}

1. Kröber T, Guerin P: An in vitro feeding assay to test acaricides for control of hard ticks. Pest Manag Sci 2007, 63:17-22.

2. de la Fuente J, Almazán C, Blouin EF, Naranjo V, Kocan KM: Reduction of tick infections with Anaplasma marginale and A. phagocytophilum by targeting the tick protective antigen subolesin. Parasitol Res 2006, 100:85-91.

3. Tajeri S, Razmi G: Hyalomma anatolicum anatolicum and Hyalomma dromedarii (Acari: Ixodidae) imbibe bovine blood in vitro by utilizing an artificial feeding system. Vet Parasitol 2011, 180:332-335.

4. Kröber T, Guerin P: The tick blood meal: from a living animal or from a silicone membrane? ALTEX 2007, Spec Issue:39-41.

5. Kröber T, Guerin P: In vitro feeding assays for hard ticks. Trends Parasitol 2007, 23:445-449.

6. Kemp D, Stone B, Binnington K: Tick Attachment and Feeding: Role of the Mouthparts, Feeding Apparatus, Salivary Gland Secretions and the Host Response. In Current Themes in Tropical Science. Volume 1. Physiology of Ticks. Edited by Obenchain FD, Galun R. Oxford: Pergamon Press Ltd; 1982:119-168

7. Fujisaki K, Kitaoka S, Morii T: Comparative observations on some bionomics of Japanese ixodid ticks under laboratory cultural conditions. Natl Inst Anim Health Q (Tokyo) 1976, 16:122-128.

8. Fujisaki K: Development of acquired resistance and precipitating antibody in rabbits experimentally infested with females of Haemaphysalis longicornis (Ixodoidea: Ixodidae). Natl Inst Anim Health Q (Tokyo) 1978, 18:27-38

9. Anisuzzamman, Islam M, Alim M, Miyoshi T, Hatta T, Yamaji K, Matsumoto Y, Fujisaki K, Tsuji N: Longistatin is an unconventional serine protease and induces protective immunity against tick infestation. Mol Biochem Parasitol 2012, 182:45-53.

10. Akov S: Blood Digestion in Ticks. In Current Themes in Tropical Science. Volume 1. Physiology of Ticks. Edited by Obenchain FD, Galun R. Oxford: Pergamon Press Ltd; 1982:197-211.

11. Oliveira MC, Oliveira-Sequeira TC, Araujo JP, Amarante AF, Oliveira HN: Babesia spp. infection in Boophilus microplus engorged females and eggs in Sao Paulo State, Brazil. Vet Parasitol 2005, 130:61-67.

12. Franta Z, Frantová H, Konvičková J, Horn M, Sojka D, Mareš M, Kopáček P. Dynamics of digestive proteolytic system during blood feeding of the hard tick Ixodes ricinus. Parasit Vectors 2010, 3:119.

13. Miyoshi T, Tsuji N, Islam M, Kamio T, Fujisaki K: Cloning and molecular characterization of a cubilin-related serine proteinase from the hard tick Haemaphysalis longicornis. Insect Biochem Mol Biol 2004, 34:799-808.

14. Boldbaatar D, Sikalizyo Sikasunge C, Battsetseg B, Xuan X, Fujisaki K: Molecular cloning and functional characterization of an aspartic protease from the hard tick Haemaphysalis longicornis. Insect Biochem Mol Biol 2006, 36:25-36.
15. Tsuji N, Miyoshi T, Battsetseg B, Matsuo T, Xuan X, Fujisaki K: A cysteine protease is critical for Babesia spp. transmission in Haemaphysalis ticks. PLOS Pathog 2008, 4:e1000062.

16. Motobu M, Tsuji N, Miyoshi T, Huang X, Islam M, Alim M, Fujisaki K: Molecular characterization of a blood-induced serine carboxypeptidase from the ixodid tick Haemaphysalis longicornis. FEBS J 2007, 274:3299-3312.

17. Alim M, Tsuji N, Miyoshi T, Islam M, Huang X, Motobu M, Fujisaki K: Characterization of asparaginyl endopeptidase, legumain induced by blood feeding in the ixodid tick Haemaphysalis longicornis. Insect Biochem Mol Biol 2007, 37:911-922.

18. Alim M, Tsuji N, Miyoshi T, Islam M, Huang X, Hatta T, Fujisaki K: HILgm2, a member of asparaginyl endopeptidases/legumains in the midgut of the ixodid tick Haemaphysalis longicornis, is involved in blood-meal digestion. J Insect Physiol 2008, 54:573-585.

19. Hatta T, Kazama K, Miyoshi T, Umemiya R, Liao M, Inoue N, Xuan X, Tsuji N, Fujisaki K: Identification and characterisation of a leucine aminopeptidase from the hard tick Haemaphysalis longicornis. Int J Parasitol 2006, 36:1123-1132.

20. Chrudimská T, Slaninová J, Rudenko N, Růžek D, Grubhoffer L: Functional characterization of two defensin isoforms of the hard tick Ixodes ricinus. Parasit Vectors 2011, 4:63.

21. Zhou J, Liao M, Ueda M, Gong H, Xuan X, Fujisaki K: Sequence characterization and expression patterns of two defensin-like antimicrobial peptides from the tick Haemaphysalis longicornis. Peptides 2007, 28:1304-1310.

22. Hatta T, Tsuji N, Miyoshi T, Alim M, Islam M, Fujisaki K: Leucine aminopeptidase in the ixodid tick Haemaphysalis longicornis: endogenous expression profiles in midgut. J Vet Med Sci 2009, 71:589-594.

23. Rodriguez-Valle M, Lew-Tabor A, Gondro C, Moolhuijzen P, Vance M, Guerrero FD, Bellgard M, Jorgensen W: Comparative microarray analysis of Rhipicephalus (Boophilus) microplus expression profiles of larvae pre-attachment and feeding adult female stages on Bos indicus and Bos taurus cattle. BMC Genomics 2010, 11:437.

24. Castelli E, Caputo V, Morello V, Tomasino RM: Local reactions to tick bites. Am J Dermatopathol 2008, 30:241-248.

25. Callow LL: The infection of Boophilus microplus with Babesia bigemina. Parasitology 1968, 58:663-670.

doi:10.1186/1756-3305-5-263

Cite this article as: Hatta et al:: Semi-artificial mouse skin membrane feeding technique for adult tick, Haemaphysalis longicornis. Parasites \& vectors 2012 5:263.

\section{Submit your next manuscript to BioMed Central and take full advantage of:}

- Convenient online submission

- Thorough peer review

- No space constraints or color figure charges

- Immediate publication on acceptance

- Inclusion in PubMed, CAS, Scopus and Google Scholar

- Research which is freely available for redistribution 